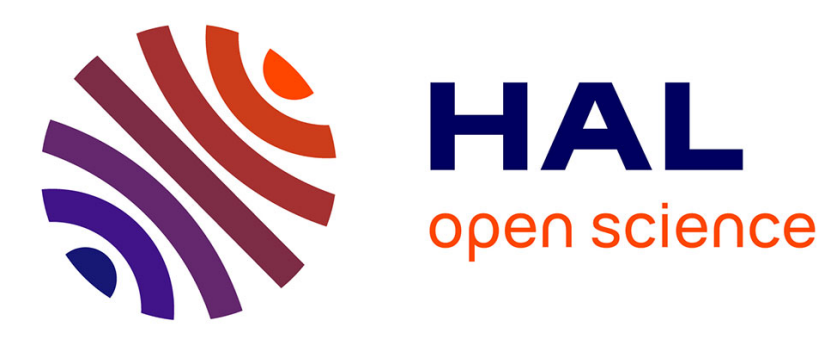

\title{
Vingt ans après La Russie et la quête de puissance
}

Marie Mendras

\section{To cite this version:}

Marie Mendras. Vingt ans après La Russie et la quête de puissance: La Russie et la quête de puissance.

Commentaire, 2011, 4 (136), pp.891 - 900. 10.3917/comm.136.0891 . hal-01024506

\section{HAL Id: hal-01024506 \\ https://hal-sciencespo.archives-ouvertes.fr/hal-01024506}

Submitted on 16 Jul 2014

HAL is a multi-disciplinary open access archive for the deposit and dissemination of scientific research documents, whether they are published or not. The documents may come from teaching and research institutions in France or abroad, or from public or private research centers.
L'archive ouverte pluridisciplinaire HAL, est destinée au dépôt et à la diffusion de documents scientifiques de niveau recherche, publiés ou non, émanant des établissements d'enseignement et de recherche français ou étrangers, des laboratoires publics ou privés. 


\title{
Vingt ans après La Russie et la quête de puissance
}

\author{
MARIE MENDRAS
}

Vingt ans après l'effondrement de l'URSS, la politique étrangère de la Russie, tout comme la politique intérieure, reste profondément marquée par la chute de 1991. Les hommes qui dirigent le pays aujourd'hui ont une vision négative de leur histoire nationale. Ils continuent à voir dans l'extraordinaire bouleversement des réformes gorbatchéviennes et de l'éclatement de l'empire une perte considérable. 1991 a été une "catastrophe géopolitique», répète Vladimir Poutine. Cette rupture brutale avec le passé laisse de profondes traces, tant dans les mentalités que dans le comportement des élites dirigeantes.

M. M.

F IDÈLE à la tradition doctrinaire soviétique, l'ancien chef du FSB (les services de renseignement) affirme la puissance russe dans une trajectoire historique - l'héritage légitime du passé - et dans un face à face avec les États-Unis - l'équilibre entre deux superpuissances, entre l'Est et l'Ouest. Il s'appuie sur les moyens classiques de la politique étrangère : croissance de l'économie nationale, renforcement des positions militaires et stratégiques, consolidation d'une sphère d'influence, rôle à l'ONU. Il sait que le moteur de la politique russe à l'avenir sera l'énergie et le commerce, et pense que la structure reste la dissuasion nucléaire et la sphère d'influence. Il bénéficie d'un atout de taille, l'exportation d'hydrocarbures au prix fort, mais fait face à de sérieux défis : la mondialisation, la puissance chinoise et la faible compétitivité de l'économie russe.
Cet article propose une lecture de la vision que les élites russes ont du monde, et de leur place dans le monde, vingt ans après 1991, en mettant l'accent sur la question de l'identité nationale et la consolidation du régime poutinien, et sur la relation complexe avec l'Europe et l'Occident, relation dans laquelle les pays du voisinage commun (Ukraine, Géorgie, Moldavie...) se trouvent aujourd'hui pris en étau entre l'Union européenne et la Russie. Cet « entre-deux » constitue pour Moscou un point de fixation qui tend à masquer l'enjeu crucial à moyen et long terme : la transformation de l'Asie et la mondialisation. Pourtant, la Chine développe rapidement ses relations avec plusieurs républiques ex-soviétiques et accroît ses investissements en Russie.

Le repli sur une sphère de proximité territoriale, tout en revendiquant des ambitions de 
puissance mondiale, nourrit l'ambivalence et la fragilité des positions russes. Cette ambivalence se reflète en politique intérieure et a pris une forme emblématique dans le «tandem» Poutine-Medvedev. De l'été 2008 à fin 2011, Dmitri Medvedev a rempli efficacement sa fonction de Président-diplomate, sans être ni le stratège ni le décideur in fine. Cependant, Vladimir Poutine a choisi de mettre fin au jeu de rôles et de se faire réélire Président en mars 2012. Il reprend toutes les prérogatives de chef d'État et chef des armées.

\section{Le mythe du « retour»}

Les verbes « redevenir", « reprendre », « retrouver» se rencontrent à l'infini dans les discours et articles russes. Retrouver le statut de grande puissance, reprendre sa place sur le continent, redevenir un État moderne et fort. De même, le discours russe préfère le préfixe «ex» ou préfixe "post». La Russie est un pays ex-soviétique, entouré de républiques ex-soviétiques et des «ex-démocraties populaires » d'Europe centrale.

Avec l'effondrement de l'URSS, la Russie a perdu son État et sa puissance, derjava. La notion de derjava est quasiment synonyme de l'État, dans une pensée russe résolument fidèle aux théories réalistes des rapports internationaux. Depuis la victoire sur l'Allemagne nazie, la puissance a été pensée et vécue comme intangible, existentielle. Le terme derjava, dérivé du verbe derjat', tenir, exprime la notion de contrôle du territoire, des ressources, des frontières. Pour Moscou, la perte du statut de grande puissance se traduit en affaiblissement de l'État et montée de l'influence des autres États. Ainsi, le thème du «retour» de puissance traduit-il le désir de récupérer un statut passé. Insistons sur l'importance accordée par les élites russes au statut plus qu'à une réelle stratégie de puissance impliquant volonté, énergie, ressources et visant à la domination. La position de l'URSS avant 1989-1991 est magnifiée et l'action présente vise à « rattraper » des positions perdues. Le regret du passé pèse plus que la réalité du présent et l'ambition d'un futur.

La réécriture de l'histoire officielle depuis quelques années révèle les sensibilités des élites poutiniennes, et de la société qu'elles gouvernent. La Grande Guerre patriotique est l'ultime monument de l'épopée soviétique. Les autres grands épisodes héroïques ont perdu leur éclat ou leur sens (1917, l'industrialisation des années 1930, l'atome, Gagarine et la conquête de l'espace). La mémoire de la victoire contre l'Allemagne nazie est préservée comme un bien précieux, source de fierté et d'identité, et socle de la puissance soviétique après 1945. L'utopie de la révolution mondiale avait été enterrée dès la fin des années 1920 et l'objectif de la puissance stratégique était devenu le ressort du régime soviétique.

Si les Russes acceptaient aujourd'hui que la quête de superpuissance et de victoire sur l'Autre (le camp capitaliste) n'avait pas de sens, comment justifier les décennies de drames et de sacrifices depuis la guerre civile dès 1918 et pendant les trente années de dictature stalinienne? C'est essentiellement pour conserver cette légitimité du sacrifice subi (et, par conséquent, l'inévitabilité des répressions commises) par les générations passées que Staline reste une figure héroïque et terrifiante dans l'historiographie poutinienne. Staline est le dictateur sanguinaire ce fait n'est pas contesté - qui a fait d'un empire humilié une superpuissance damant le pion à l'Amérique et à la vieille Europe. Il a bâti, conquis, dominé, alors que Mikhaïl Gorbatchev et Boris Eltsine ont détruit. La plupart des manuels d'histoire publiés ces dernières années véhiculent cette apologie des périodes de «puissance » contrastant avec les régimes condamnables qui ont laissé la Russie s'affaiblir $\left({ }^{1}\right)$.

Quand la Russie s'est ouverte et rapprochée de l'Europe et de l'Occident, pendant la perestroika de Mikhail Gorbatchev puis les réformes de Boris Eltsine, elle a perdu l'aura de la puissance déjà sur le déclin. Ce déclin était en cours depuis les années 1970 mais en grande partie occulté à Moscou et minimisé dans les capitales occidentales. Aux yeux des Russes, l'ouverture et la réforme des années 1986-1993 sont responsables de la terrible crise qui s'ensuit $\left(^{2}\right)$. Alors que les Occiden-

(1) Lidéologie véhiculée par les manuels d'histoire et les films documentaires est analysée par les historiens de l'association Mémorial, notamment Arseni Roguinski. Entretiens avec l'auteur à Moscou et à Paris en 2009 et 2010.

(2) Voir les enquêtes d'opinion du Centre Levada d'étude de l'opinion publique, www.levada.ru. 
taux se réjouissaient de la «libération » des Soviétiques et de la victoire de la démocratie, la grande majorité des Russes vivaient un traumatisme majeur auquel ils n'étaient pas préparés.

Il est important de souligner le décalage entre le vécu russe et notre vision occidentale des années gorbatchéviennes et de la fin du communisme. Nous avons observé avec enthousiasme et bonne conscience cette période de défaite de l'URSS dans la guerre froide et d'enchantement du politique grâce à la «libération» des populations enfermées dans le soviétisme. Ce grand malentendu entre les Russes et nous sur l'épisode fondamental de leur histoire récente marquera pendant encore longtemps notre relation avec eux. La ligne de démarcation politique et mémorielle entre l'Europe et la Russie a bougé mais elle reste une ligne Est-Ouest.

La société russe a, dans l'ensemble, très mal vécu les années 1990 et en conçoit une hostilité au changement et à l'internationalisation, et un profond conservatisme. Vladimir Poutine a profité de cet état d'esprit et a bâti son système sur l'ordre social sans la démocratie politique, la croissance économique sans les réformes, et l'image d'une Russie plus forte à l'étranger $\left({ }^{3}\right)$.

« La Russie n'est pas tant un pays émergent qu'une ancienne superpuissance qui a échoué $\left({ }^{4}\right)$ », résume l'économiste finlandais Pekka Sutela. Les contradictions du régime poutinien se nouent dans cette difficulté à lire l'histoire récente avec recul et à lâcher des positions qui n'apportent plus d'avantages. "Bygones are bygones» est la règle d'or des économistes. Ce qui est perdu est perdu : ne pas continuer d'investir dans un puits sans fond, ne pas s'arrimer à des positions qui appartiennent au passé. Les hommes politiques ont souvent tendance à défendre les acquis et colmater les brèches; ils hésitent à prendre le risque d'une approche radicalement nouvelle. Ils «tiennent» plus qu'ils ne construisent.

Ce réflexe, plus prononcé chez les dirigeants russes, explique le prolongement d'une conception protectionniste, presque négative,

(3) Voir Marie Mendras, Russie. L'envers du pouvoir, Odile Jacob, 2008.

(4) Pekka Sutela, «How strong is Russia's economic foundation? ", Center for European Reform, Policy Brief, octobre 2009, p. 1 (www.cer.org.uk). de la relation avec l'extérieur, même avec les voisins proches, dévoilant le manque de confiance dans un projet national. "Les passions existent au niveau des nations, mais elles tendent souvent à être surtout défensives et négatives ", observe Pierre Hassner $\left(^{5}\right)$. La politique internationale de la Russie porte en elle émotions, regrets et volonté de prendre une revanche sur les années noires.

Est-ce un objectif raisonnable, pour Moscou, de chercher à revenir à une position de puissance déterminée par la guerre froide alors que le monde s'est transformé en profondeur et que des acteurs clés, comme la Chine, opèrent des mutations incroyablement plus rapides que la Russie ? Lespace et le temps ne sont plus des atouts pour un vaste pays qui parle beaucoup de modernisation mais peine à se développer au même rythme que ses voisins ${ }^{6}$ ).

L'empire tsariste a-t-il jamais été une puissance dominante? Il a vaincu l'armée de Napoléon, est entré dans le concert des nations au Congrès de Vienne en 1815, s'est agrandi et a noué des alliances tout au long du XIX ${ }^{\mathrm{e}}$ siècle, mais a également subi de graves revers - la guerre de Crimée en 1856, la défaite face au Japon en 1905 - et s'est décomposé pendant la Première Guerre mondiale. De 1918 à 1941, la Russie soviétique vit quasiment en autarcie, coupée de l'Europe et de l'Asie. Linvasion allemande de juin 1941 plonge la dictature stalinienne dans les affaires du monde. La satellisation de l'Europe centrale et les années de guerre froide et d' «équilibre de la terreur» (dissuasion nucléaire), de Khrouchtchev à Brejnev, constituent la période la plus «internationale » de l'histoire russe. Ces trois décennies de «superpuissance » ne sont-elles pas l'exception plutôt que la règle? Aucun expert russe n'oserait poser une question aussi iconoclaste. Même des politologues et experts critiques de l'autoritarisme poutinien acceptent le postulat d'une Russie naturellement et nécessairement grande puissance $\left({ }^{7}\right)$. De manière anachronique, le discours russe se décline principale-

(5) Pierre Hassner, préface in Philippe Esper et al., Un monde sans Europe?, Fayard, 2011, p. 30.

(6) Pierre Buhler, La Puissance au XXI siècle. Les nouvelles définitions du monde, CNRS Éditions, 2011, p. 177-186.

(7) Le travail de réflexion, «Russie. Scénarios 2020 », mené par le Centre Carnegie de Moscou en 2011, reflète cet état d'esprit. Plusieurs études sont consacrées à la politique étrangère russe. Même Thomas Graham, diplomate américain russophone, fonde 
ment autour des notions de "défense de la souveraineté nationale » et de "sphère d'intérêts privilégiés », comme si cet invariant d'une grande Russie était la perspective la plus stabilisante.

Dmitri Trenin, directeur du Centre Carnegie à Moscou, œuvre pour le rétablissement d'une Russie forte. Mais il souligne fort justement que le premier défi est de garder le contrôle sur la Sibérie et l'Extrême-Orient, et de rétablir la sécurité dans les républiques du Nord-Caucase. Le problème est donc d'abord un problème de cohésion interne, "l'intégration au centre », selon la formule de Trenin, et d'utilisation judicieuse des ressources économiques et territoriales nationales $\left(^{8}\right)$.

Pour Fedor Lukyanov, rédacteur en chef de la revue Russia in Global Affairs, le conflit d'août 2008 en Géorgie est venu confirmer que la politique étrangère russe n'avait plus besoin de se focaliser sur l'Occident et qu'il était temps pour l'OTAN de ne pas transgresser la «ligne rouge ", celle de la «sphère des intérêts privilégiés » de la Russie dans les républiques ex-soviétiques voisines de l'Europe. Dans ses éditoriaux, Lukyanov revient régulièrement sur « la fermeture de la parenthèse » des années 1990 et le retour à l'équilibre des puissances $\left({ }^{9}\right)$.

Ces analyses engagées reposent sur un raisonnement fragile car elles n'indiquent pas par quels moyens et avec quelles méthodes, et dans quel but, la Russie rebâtirait un grand espace militaire et économique tenant tête à l'Occident d'un côté, à la Chine et à l'Asie de l'autre. Elles sont contestées par des chercheurs russes indépendants qui soulignent au contraire la nécessité pour leur pays de s'adapter aux nouvelles réalités mondiales et de ne pas sacrifier l'indispensable modernisation à la vaine quête d'une puissance disparue. Yuri Fedorov s'inquiète d'une relance de la politique d'armement en Russie et d'un nouveau face à face sécuritaire sur le conti-

son raisonnement sur le fait que «la Russie a été une grande puissance pendant trois cents ans » et qu' «elle a regagné pour une bonne part son statut de puissance majeure ». Il se félicite que la Russie reste « la puissance dominante dans l'espace ex-soviétique » et devienne « un pilier fondamental dans la nouvelle architecture de sécurité européenne, aux côtés de l'OTAN et de l'Union européenne ». Ces travaux sont en cours de publication dans la revue en russe Pro et Contra, Moscou. Voir aussi le site www.carnegie.ru.

(8) Dmitri Trenin, Post-Imperium, Carnegie Endowment for Peace, Washington, DC, 2011.

(9) Revue trimestrielle publiée en russe et en anglais, Russia in Global Affairs, Moscou. nent européen $\left({ }^{10}\right)$. Lilia Chevtsova dénonce l'utilisation des thèmes anti-occidentaux et la stratégie dominatrice de Moscou dans les pays du voisinage commun, Géorgie et Ukraine. Elle souligne les lourdes conséquences de l'impuissance du régime eltsinien tant sur l'évolution intérieure que sur la politique étrangère encore aujourd'hui $\left({ }^{11}\right)$.

En effet, pendant les années Eltsine, la Russie n'a pas forgé de politique étrangère. Elle était trop occupée par la reconstruction intérieure dans un contexte de chute économique et sociale $\left({ }^{12}\right)$. Elle suivait le mouvement vers l'ouverture, la coopération avec l'Europe, les États-Unis et l'OTAN. Elle dépendait de la bonne volonté des anciens rivaux pour sauver son économie, son armée, son intégrité territoriale. L'État russe a fini par échouer sur le désastre de la guerre en Tchétchénie et le krach financier d'août 1998. L'humiliation était totale. Les frappes aériennes de l'OTAN sur la Serbie pour défendre le Kosovo au printemps 1999 ont choqué les Russes et sonné le réveil de la politique étrangère.

Dans la première moitié des années 2000, l'équipe poutinienne a reconstruit une politique internationale sur la force énergétique, grâce à l'envolée des prix depuis 2001, et par la relance d'un partenariat stratégique avec les États-Unis après les attentats du 11 septembre 2001. Sans les considérables ressources en pétrole, gaz naturel, et autres matières premières, et sans la «guerre " commune contre le terrorisme, la question d'un « retour» de la puissance russe ne se serait même pas posée. L'arsenal nucléaire n'aurait pas suffi à assurer à Moscou une position de force sur la scène internationale.

La seconde moitié des années 2000 se différencie de la première période Poutine. En effet, l'année 2004 met le Président russe devant des défis majeurs en Russie et dans son «étranger proche», terme par lequel les Russes dénomment leurs anciennes républiques dont ils contestent la pleine souverai-

(10) Yuri Fedorov, «The Russia factor in international security. An assesssment of Moscow's ambitions and capacity ", étude présentée au séminaire de l'Observatoire de la Russie, CERI/Sciences Po, 6 juillet 2011.

(11) Lilia Shevtsova, Lonely Power, Carnegie Endowment of Peace, Washington DC, 2010, et «Politics inside, policy outside. How russian elites preserve the status quo and look modern ", étude présentée au séminaire de l'Observatoire de la Russie, CERI/Sciences Po, 28 septembre 2011.

(12) Marie Mendras, « La Russie en mal de politique étrangère », Pouvoirs, n 88, 1999, p. 107-120. 
neté. La deuxième guerre de Tchétchénie a accru l'insécurité et la violence dans tout le Nord-Caucase. Vladimir Poutine est pris de court devant les révolutions de couleur en Géorgie et en Ukraine, en 2003 et 2004 ; il craint la contagion démocratique en Russie. Il mettra alors tout en œuvre pour affaiblir les nouveaux régimes, augmenter la présence économique russe, attiser les conflits territoriaux en Géorgie.

Sa stratégie visera essentiellement à arrêter l'élargissement de l'OTAN et à tenir les pays de l'entre-deux hors de la sphère occidentale, menant à la guerre en Géorgie en août 2008. La Russie brave un interdit : elle envoie son armée combattre sur le territoire d'un État indépendant membre de l'ONU et reconnaît l'indépendance des provinces d'Abkhazie et d'Ossétie du Sud $\left({ }^{13}\right)$. Les militaires russes occupent toujours, en 2011, des parties de la Géorgie. Le raidissement de la politique russe aux portes de l'OTAN et de l'Union européenne, parallèlement au durcissement du régime Poutine contre la société civile et l'opposition, et à l'accroissement de la corruption au sommet du pouvoir aura pour effet de freiner le rapprochement avec Washington, le fameux reset lancé par Barack Obama en 2009, marqué notamment par la signature du nouvel accord START, traité de réduction des armes nucléaires, en avril 2010.

\section{L’identité nationale et l'image de l'ennemi}

Vladimir Poutine a convaincu les Russes du fait que, sans un regain de puissance sur les autres États, leur pays était en danger. De nouveau depuis 2000 , le paradigme de l'ennemi intérieur et extérieur, de la patrie en danger, occupe le cour de la doctrine et s'insinue dans les mentalités. La guerre en Géorgie en août 2008 n'était rendue possible, et acceptable par la population russe, qu'avec cette insécurité dans les têtes. Les élites russes ont dès 19931994 douté des bonnes intentions de leurs voisins et partenaires et adhéré à une matrice d'explication toute en défiance : l'Ouest tenait à maintenir leur pays affaibli pour construire un système international unipolaire, dominé par

(13) Ronald Asmus, A Little War that Shook the World: Georgia Russia, and the Future of the West, 2010, Roy Allison, « Russia resurgent ? Moscow's campaign to "coerce Georgia to peace" ", International Affairs, vol. 84, $\mathrm{n}^{\circ}$ 6, novembre 2008, p. 1145-1171.
l'Amérique. Étonnamment, la montée de la Chine, de l'Inde, du Brésil, qui a remis en question le modèle unipolaire, n'a pas corrigé de manière significative cette lecture déformée des intentions des pays occidentaux et de leurs organisations (OTAN, Union européenne).

Dans son discours à Munich en février 2007, Vladimir Poutine attaque frontalement la politique «unilatérale» américaine et les projets d'élargissement de l'OTAN, dénonçant une attitude de guerre froide et un danger pour la sécurité de la Russie. «Les États-Unis ont franchi les limites de leurs frontières nationales [...] Plus personne ne se sent en sécurité $\left({ }^{14}\right)$. »

Lun des messages que fait passer Poutine est que la Russie défend d'abord et avant tout ses intérêts, dans un monde où les autres États ne pensent aussi qu'à défendre leurs intérêts. Derrière un discours de coopération, les leaders occidentaux auraient un agenda caché. L'Union européenne reste, pour Poutine, une construction mystérieuse, qui ne peut être gouvernée démocratiquement et équitablement, et qui est en réalité contrôlée par les grands États et les grands groupes industriels et financiers. Lui est-il possible de concevoir qu'un dirigeant puisse privilégier un effort sur le long terme alors qu'il ne sera plus au pouvoir pour bénéficier des profits de sa stratégie? Et d'imaginer qu'un Parlement ou une Cour ait vraiment autorité pour contrer les décisions du Chef dans le domaine régalien de la politique étrangère? Quand la justice britannique a refusé d'extrader l'ancien ministre tchétchène Akhmed Zakaïev au début des années 2000, Vladimir Poutine a fait comprendre au Premier ministre Tony Blair qu'il le jugeait responsable du refus puisque cette affaire était, du point de vue russe, hautement politique. Interrogé sur les élections «contrôlées » chez lui, il répondait que George W. Bush avait été mal élu. Questionné sur la Tchétchénie, il faisait immédiatement l'amalgame avec Al-Qaïda (15). Lattitude des dirigeants russes n'est compréhensible qu'en intégrant leur perception, en partie erronée, du comportement de nos gouvernements démocratiques.

(14) Discours de Vladimir Poutine, Président de Russie, à la $43^{\mathrm{e}}$ conférence sur la sécurité, Munich, 10 février 2007, www.securityconference.de/archive.

(15) Rencontres du Club Valdaï en Russie, septembre 2004 et 2005, auxquelles l'auteur a assisté. 
La tonalité anti-occidentale a baissé depuis la crise mondiale de 2008 et grâce à la présidence Medvedev. Cependant, même les propos plus amènes tenus par Dmitri Medvedev pendant ses quatre années de présidence ne sont jamais dénués d'une ou deux incises sur l'ambition militaire américaine ou la duperie européenne $\left({ }^{16}\right)$. Lidée que les pays de l'OTAN ont intérêt à freiner le "retour » de la puissance russe est bien ancrée dans les mentalités. Cela révèle un attachement à la notion classique de la puissance dans laquelle la force et le statut d' "égal rival» comptent plus que le partenariat et la mise en commun des moyens et des finalités de la stratégie. La réticence à partager dévoile la crainte d'exposer les arcanes du système politique et économique russe et de le vulnérabiliser.

Dans la plupart des négociations multilatérales, les responsables russes ont le souci de cloisonner les sujets et d'éviter le lien entre sécurité militaire et droits de l'Homme, entre sécurité énergétique et transparence commerciale. Les préparatifs du sommet de l'OSCE à Astana en décembre 2010 en sont un bon exemple $\left({ }^{17}\right)$. Le refus russe de respecter la Charte de l'énergie, qu'elle a signée, fournit un autre exemple de primauté de la «souveraineté nationale » sur les engagements multilatéraux.

Pourtant, la paix russe est d'abord menacée de l'intérieur du système de pouvoir ; le principal danger contre la stabilité de l'État et la tranquillité des populations vient sans aucun doute d'une politique brutale et inefficace menée en Tchétchénie. Le terrorisme et la violence extrême ont trouvé un terreau fertile dans les deux guerres menées par l'armée russe $\left({ }^{18}\right)$ et dans la pauvreté qui frappe les populations caucasiennes. Andrei Soldatov et Irina Borogan démontrent que les services de renseignement et les siloviki (hommes des ministères de force) assurent la protection des intérêts des organisations et des clans qui forment la structure du régime et jouent un rôle démesuré dans la prise de décision arbitrée par Vladimir Poutine $\left({ }^{19}\right)$. La sécurité

(16) Voir par exemple son entretien télévisé du 16 mai 2011 et son entretien au quotidien Izvestiia le 4 août 2011, www.kremlin.ru.

(17) Conférence de réflexion après le sommet d'Astana, OSCE, Vienne, 13 décembre 2010.

(18) Sur la deuxième guerre en Tchétchénie, lire Anna Politkovskaïa, Tchétchénie. Le déshonneur russe, Buchet/Chastel, 2003.

(19) Andrei Soldatov, Irina Borogan, Les Héritiers du KGB, François Bourin Éditeur, 2011. nationale est d'abord la sécurité de l'État et du régime, et non la sécurité des personnes. Sans aucun doute, le fait que Dmitri Medvedev rende à son mentor le fauteuil du Kremlin en 2012 indique une politique étrangère moins conciliante, conduite en grande partie par les proches de Poutine, qui ont appartenu aux services de renseignement et contrôlent les grands monopoles et holdings financiers, comme Igor Setchine ou Serguei Ivanov.

Le thème de l'ennemi intérieur a tragiquement retrouvé sa place de choix dans le discours officiel : l'ennemi tchétchène, le terroriste islamiste, les Caucasiens "portés à la violence », les «extrémistes ». Pour une bonne part, le conflit d'août 2008 avec la Géorgie résulte du drame tchétchène et de l'atmosphère de "nation en danger » entretenue par le régime russe. Le Président Mikheil Saakachvili est «l'ennemi extérieur» à abattre, comme n'hésite pas à le clamer le Président Medvedev au troisième anniversaire de la guerre $\left({ }^{20}\right)$. Ainsi, l'idée selon laquelle Medvedev n'aurait pas approuvé l'intervention en 2008 et chercherait trois ans plus tard à se démarquer de la ligne dure de Poutine ne tient pas. Medvedev a au contraire utilisé cet épisode pour se donner une image de ferme patriote et tenter de gagner, en vain, un second mandat présidentiel en 2012.

Le sociologue Lev Goudkov observe très justement que la Russie est « un pays nationaliste sans idée nationale $\left({ }^{21}\right) »$. Le Russe ordinaire, explique Goudkov, existe contre. Il s'identifie à la communauté nationale par défaut : «je ne suis ni géorgien, ni juif, ni européen ». Il regrette la puissance soviétique perdue en ce qu'elle lui apportait une identité forte et l'assurance d'appartenir à un tout bien défini et respecté. Aujourd'hui, il n'a pas retrouvé ce sentiment d'appartenance car la Fédération de Russie est un espace politique, économique et social de plus en plus distendu, disparate, mal gouverné, corrompu, où les régions communiquent peu entre elles et où les classes sociales se côtoient à peine ${ }^{(22}$.

(20) Entretien avec Dmitri Medvedev, Izvestiia, 4 août 2011

(21) Lev Goudkov, Negativnaia identitchnost' [L'identité négative], Moscou, Novoe Literatournoe Obozrenie, 2004, et Abortnaia demokratiia [La démocratie avortée]; Moscou, Rosspen, 2011.

(22) Voir les travaux du Centre Levada, publiés dans le Monitoring Obchtchestvennogo mneniia, et sur le site levada.ru., et les travaux de l'équipe du démographe Anatoli Vichnevski, demoscope.ru. 
Vladimir Poutine a encouragé cette fragmentation de l'espace et cette dissolution du lien social par une politique systématique d'affaiblissement des institutions publiques au profit de la domination des réseaux et grands groupes financiers qu'il contrôle. Plus le pouvoir personnel et clientéliste domine, plus l'État s'affaiblit ( $\left.{ }^{23}\right)$. Poutine a ainsi paradoxalement fragilisé ce qui fait la base d'une stratégie de puissance classique : l'État national fort, homogène, solidaire, souverain. Et il n'a pas construit de système d'alliances ou de communauté économique dynamique.

En cultivant ces images de l'ennemi et cette profonde méfiance des négociations multilatérales, en préférant des accords bilatéraux reposant sur des relations de dirigeant à dirigeant, les gouvernants russes se privent d'alliés et de partenaires fiables. Certaines anciennes républiques ont contracté des accords militaires avec Moscou, mais l'Organisation du traité de sécurité collective $\left({ }^{24}\right)$ ne fournit pas la base d'une défense commune. Le projet d'union douanière rassemblant la Russie, la Biélorussie, le Kazakhstan et bientôt le Kirghistan, reste modeste. La Communauté des États indépendants, formée à la va-vite dans la tourmente de décembre 1991, demeure un ensemble faiblement structuré et dont la trame est d'abord faite d'accords bilatéraux entre Moscou et chacune des capitales concernées.

\section{Les pays de l'entre-deux, pris entre l'Est et l'Ouest}

Plus que tout, Vladimir Poutine craint les perturbations politiques dans son «étranger proche » et la contagion démocratique qu'elles peuvent provoquer en Russie même. Il a nié la réalité de la révolte démocratique à Kiev en 2004, affirmant que c'était une manipulation américaine. Il a ensuite tout mis en ouvre pour affaiblir le nouveau gouvernement.

(23) Cette analyse est développée par l'auteur dans son ouvrage à paraître en 2012 : Russian Politics. The Paradox of a Weak State, Hurst, Londres et Columbia University Press, New York.

(24) En décembre 1991 est formée la Communauté des États indépendants autour de la Russie, l'Ukraine, la Biélorussie et le Kazakhstan, que rejoindront l'Arménie, l'Azerbaïdjan, les républiques d'Asie centrale. La Géorgie appartient à la CEI de 1998 à 2008. L'Organisation de sécurité réunit aujourd'hui, autour de la Russie, la Biélorussie, l'Arménie et les États d'Asie centrale, excepté le Turkmenistan.
Le contrôle sur les régimes politiques et économies voisins est absolument crucial à la survie du régime poutinien. Or, ce contrôle est facilité par la prudence des politiques occidentales depuis 2008. Notre prudence s'explique à la fois par le souci de maintenir un partenariat stratégique et énergétique avec la Russie, et par les évolutions politiques peu prometteuses en Ukraine, Biélorussie et Azerbaïdjan notamment.

En Europe, nous désignons les pays pris en sandwich entre nous et la Russie par les termes de « voisinage commun » et de « partenariat oriental ». En Russie, on parle de l'exUnion (byvchii Soiuz) et de «l'étranger proche ». La nuance est de taille. Les dirigeants russes tiennent à démontrer que la souveraineté nationale des pays voisins est fragile et qu'elle dépend largement de la bonne relation avec Moscou. Nourrie de propagande sur les errements de la Géorgie, de l'Ukraine, et même de l'Estonie, membre de l'Union européenne, la population russe tend à percevoir ces pays comme ayant encore un rapport particulier avec la Russie, ce qui n'en fait pas des pays totalement indépendants. Les sondages d'opinion sont révélateurs. À la question, «la Biélorussie est-elle un pays étranger? », près des deux tiers des personnes interrogées répondent négativement $\left({ }^{25}\right)$.

Les pays du Caucase constituent le point névralgique de la politique russe dans l'espace ex-soviétique. Les chars russes postés en Ossétie sont à moins de $50 \mathrm{~km}$ de Tbilissi. Poutine n'a pas atteint tous ses objectifs en choisissant le conflit avec la Géorgie en 2008. Le Président Saakachvili reste en place et les tentatives de fomenter un coup d'Etat pour le renverser ont échoué. Aucun pays ex-soviétique n'a reconnu l'indépendance de l'Abkhazie et de l'Ossétie du Sud.

Les trois pays du Caucase - Géorgie, Arménie, Azerbaïdjan - se trouvent dans une position instable. Comme les autres États de l'entre-deux, ils n'appartiennent ni à une alliance russe ni à l'UE ou l'OTAN. Les relations entre les trois États restent tendues, tout particulièrement entre l'Arménie et l'Azerbaïdjan qui n'ont pas progressé sur la voie d'un règlement politique du statut du Haut-

(25) Sondage du Centre Levada, 2007, www.levada.ru 
Karabakh, enclave peuplée d'Arméniens en Azerbaïdjan. Les relations avec Moscou sont exécrables pour la Géorgie, conflictuelles pour l'Azerbaïdjan et tendues pour l'Arménie. De l'avis de Thomas de Wahl, «en 2020, ces pays auront des liens encore plus distendus avec la Russie » $\left({ }^{26}\right)$ car les Russes sont désormais très peu nombreux dans les trois pays.

La violation de la souveraineté nationale de la Géorgie par l'armée russe, puis par le Parlement russe qui reconnaît l'indépendance de l'Abkhazie et de l'Ossétie du Sud, marque une rupture majeure, comme si Moscou tentait de revenir brutalement près d'un siècle en arrière au temps de la reconquête bolchévique.

Comme avec l'Ukraine, la Biélorussie et la Moldavie, la position du Kremlin est claire depuis la guerre de 2008 : la ligne rouge à ne pas franchir est l'adhésion de l'un des pays de l'entre-deux à une organisation dont la Russie n'est pas membre (UE, OTAN notamment). La Russie veut en quelque sorte geler les pays $\mathrm{du}$ partenariat oriental dans une position de souveraineté faible, leur refusant toute appartenance à une alliance. Les «guerres du gaz » qui empoisonnent les relations de Moscou avec Kiev depuis 2005 sont un moyen de prouver que l'Europe ne gagnera rien à s'entendre avec les pays de l'entre-deux sans passer par Moscou. La condamnation de l'ancien Premier ministre Ioulia Timochenko par le régime Ianoukovitch "pour avoir signé un accord défavorable avec la Russie » montre à quel point le commerce de l'énergie constitue un enjeu clé pour toutes les parties.

\section{La crainte de la contagion démocratique}

Moscou se replie stratégiquement sur sa région, non pas faute de mieux mais parce qu'elle fait un choix anachronique : consolider ses positions en conservant des zones tampons et en dressant des barrières entre ces pays tampons et le monde européen, alors que l'Ukraine, la Géorgie, la Moldavie devraient être des ponts entre la Russie et l'Europe. Les élites au pouvoir en Ukraine, Biélorussie, Azerbaïdjan, Arménie, acceptent cet état de fait, tout en donnant fréquemment des coups de griffe dans le «contrat tacite» avec

(26) Thomas de Wahl, contribution au projet « Russie. Scénarios pour $2020 »$, Centre Carnegie, Moscou, www.carnegie.ru.
Moscou par des appels à l'Europe, à la Turquie, à la communauté internationale. Le processus vertueux de la démocratisation/ européanisation a marqué le pas pour un temps. Limportant pour l'Europe est d'éviter un durcissement des régimes politiques à Kiev, Minsk, Moscou, et une nouvelle coupure Est-Ouest.

Pour leur part, les dirigeants russes sont inquiets et démunis devant les vagues populaires au Moyen-Orient. Ils ont observé médusés la chute de trois vieilles dictatures, en Tunisie, Égypte, Libye. Ils savent que la Syrie et d'autres pays connaîtront vraisemblablement des dénouements analogues mais ils ne réussissent pas à adapter leur politique. $\mathrm{La}$ position russe sur la Libye au Conseil de sécurité reste une exception. Le 17 mars 2011, Moscou s'abstient de mettre son veto à la résolution 1973 permettant des frappes aériennes contre le régime Kadhafi (Pékin fait de même). Début octobre, la Russie et la Chine s'opposent à la résolution prévoyant des sanctions contre la Syrie. Rappelons que précédemment la Russie avait empêché le vote de sanctions contre la Birmanie et le Soudan.

Lexception libyenne peut s'expliquer par plusieurs erreurs de jugement de la part de Moscou : la Libye ne paraissait pas cruciale, même si la Russie y négociait d'importants contrats ; les Américains resteraient en dehors de la main; les frappes aériennes ne seraient pas efficaces; il était intéressant d'avoir une attitude de bon partenaire (good stakeholder) au Conseil de sécurité. Enfin, le facteur essentiel qui a contribué à brouiller le jugement des dirigeants russes est la peur des révoltes populaires et la crainte d'évolutions similaires en Russie.

«Lextraordinaire nervosité des dirigeants russes et chinois devant les révolutions arabes, leurs mesures préventives et leurs dénonciations de ce qu'ils désignent du nom de subversion ou de croisade montrent bien qu'on exagère leur stabilité et leur assurance, et que le spectre de la démocratie continue à les hanter $\left({ }^{27}\right)$. » Pierre Hassner a raison de souligner que, dans le monde ouvert et très compétitif du XXI ${ }^{\mathrm{e}}$ siècle, les autocraties repliées sur les intérêts de quelques clans craignent plus que tout l'ouverture de leur société.

Vingt ans après la fin de l'URSS, les orien-

(27) Pierre Hassner, art. cité, p. 22. 
tations en stratégie internationale, telles qu'elles sont affichées par Poutine et ses conseillers, restent hésitantes et parfois contradictoires. Les élites russes ressentent cette incertitude et doutent de la capacité de leurs dirigeants à relever les défis économiques, technologiques, militaires des années 2020 et 2030. Plus elles doutent, plus elles adhèrent publiquement au « retour de puissance ", mais moins elles y croient. Derrière la façade d'un consensus souverainiste, les dirigeants et entrepreneurs russes sont engagés dans des joint ventures industriels et technologiques, recherchent des investisseurs, prennent part à des projets innovants à l'étranger, tentent de développer des échanges commerciaux plus lucratifs. Les exportations d'hydrocarbures et les ventes d'armes ne suffiront plus à assurer la puissance économique $\left({ }^{28}\right)$. Après avoir longtemps freiné le processus, la Russie finira pas entrer dans l'Organisation mondiale du commerce.

Le grand flou dans la pensée stratégique russe concerne la Chine et, au-delà de la Chine, l'Asie, jeune, productrice, compétitive et en plein essor financier, et qui investit en Amérique latine, en Afrique, au MoyenOrient. Un universitaire russe pose clairement

(28) Les spécialistes des questions énergétiques s'accordent sur un blocage de la stratégie russe d'ici cinq à dix ans. Lindustrie du gaz naturel est contrôlée par le géant Gazprom, géré de façon opaque et en quête d'investissements lourds pour développer les nouveaux champs gaziers et les nouvelles sources d'énergie. Voir les analyses de Mikhail Korchemkin et de Thane Gustafson. le défi : «La nécessité pour la Russie de s'assurer une présence durable dans la région Asie-Pacifique - une région clé dans le monde $\mathrm{du} \mathrm{XXI}^{\mathrm{e}}$ siècle - est incontournable. Le problème central pour la Russie aujourd'hui consiste à ne pas en devenir un simple satellite. En d'autres termes, l'évidente faiblesse de nos positions actuelles dans la région AsiePacifique devrait être compensée par une politique active de diversification optimale de nos capacités économiques et politiques $\left({ }^{29}\right)$. »

Dans dix à quinze ans, la position régionale et mondiale de la Russie sera autre, probablement moins dominatrice et plus négociatrice, certainement mieux intégrée - en matière de sécurité et d'intégration économique - à un grand ensemble continental européen qui s'ouvrira aussi aux pays de l'«entre-deux », de l'Ukraine aux pays du Caucase. La Russie en tant qu'État sera-t-elle « une grande puissance» en 2025 ou 2030 ? La question n'aura alors guère de sens car l'essentiel pour l'ancienne superpuissance, comme pour l'Europe, sera d'exister en toute sécurité, de prospérer, de compter dans les grands enjeux mondiaux, de proposer des solutions aux défis communs. Et la capacité de saisir les opportunités dépendra avant tout de la volonté politique à Moscou.

MARIE MENDRAS

(29) Dmitry Yefremenko, "Forced or desired modernity? ", Russia in Global Affairs, vol. 8, n 3, juillet-septembre 2010, p. 38 . 


\section{UN PRINCIPE JUSTE}

Il faut avouer que le principe du Parlement britannique de considérer le discours du Trône du Roi comme l'œuvre de son ministre (car il serait contraire à la dignité d'un monarque de se laisser reprocher des erreurs, de l'ignorance ou de l'inexactitude alors que la Chambre de son côté doit avoir le droit de juger du contenu du discours, de l'examiner et de le combattre), que ce principe, dis-je, est imaginé avec beaucoup de finesse et de justesse. De même aussi le choix de certaines doctrines que le gouvernement sanctionne exclusivement pour l'exposé public doit rester soumis à l'examen des savants parce qu'il ne doit pas être considéré comme étant le fait du monarque, mais d'un fonctionnaire désigné pour cela, duquel on suppose qu'il pourrait bien n'avoir pas compris la volonté de son maître ou encore l'avoir dénaturée.

Emmanuel KANT, Le Conflit des facultés (trad. Gibelin), section I, Vrin, 1973. 